\title{
Inverted (hobnail) high-grade prostatic intraepithelial neoplasia and invasive inverted pattern
}

\author{
MELTEM ÖZNUR ${ }^{1}$, SEVIM BAYKAL KOCA ${ }^{2}$, PELIN YILDIZ $^{3}$, BURAK BAHADIR ${ }^{4}$ and KEMAL BEHZATOĞLU ${ }^{5}$ \\ ${ }^{1}$ Department of Pathology, Medical Faculty, Namık Kemal University, Tekirdağ 59100; ${ }^{2}$ Department of Pathology, \\ Patnos Hospital, Ağrı 04001; ${ }^{3}$ Department of Pathology, Medical Faculty, Bezmialem University, Istanbul 34093; \\ ${ }^{4}$ Department of Pathology, Medical Faculty, Bülent Ecevit University, Zonguldak 67100; ${ }^{5}$ Department of Pathology, \\ Istanbul Education and Research Hospital, Istanbul 34098, Turkey
}

Received September 28, 2014; Accepted May 15, 2015

DOI: $10.3892 / 01.2015 .3584$

\begin{abstract}
High-grade prostatic intraepithelial neoplasia (HGPIN) is considered to be an important precursor for prostatic adenocarcinoma. The present study aimed to investigate the histological features of the uncommon inverted (hobnail) pattern of HGPIN in transrectal ultrasonographic (TRUS) prostatic needle biopsies from 13 cases. These 13 diagnosed cases of inverted HGPIN were identified out of a total of 2,034 TRUS biopsies (0.63\%), obtained from patients suspected to have prostate cancer. The hobnail pattern is comprised of secretory cell nuclei, which are histologically localized at the luminal surface of the prostate gland, rather than the periphery, and exhibit reverse polarity. Histological examinations were performed and the results demonstrated that 5 of the 13 cases exhibited pure inverted histology, while HGPIN was observed to be histologically associated with other patterns in the remaining 8 patients. In addition, an association with adenocarcinoma was identified in 7 of the 13 cases. All 7 carcinomas accompanied by inverted HGPIN were conventional acinar adenocarcinoma cases; of note, for these 7 cases, the Gleason score was 7 for each. One acinar adenocarcinoma case accompanying inverted HGPIN demonstrated hobnail characteristics in large areas of the invasive component. It was observed that nuclei were proliferated in the invasive cribriform glands, which was comparable to that of inverted HGPIN, and were located on the cytoplasmic luminal surface; a similar morphology was also observed in individual glands. In conclusion, the results of the present study suggested that the hobnail HGPIN pattern may be of diagnostic importance due to its high association
\end{abstract}

Correspondence to: Professor Kemal Behzatoğlu, Department of Pathology, Istanbul Education and Research Hospital, 12 Samatya Street, Istanbul 34098, Turkey

E-mail: kbehzatoglu@hotmail.com

Key words: inverted, hobnail, prostatic intraepithelial neoplasia, prostate, adenocarcinoma with adenocarcinoma and the high Gleason scores in the accompanying carcinomas.

\section{Introduction}

Intraepithelial neoplasia describes the atypical proliferations observed in the squamous epithelium, such as in the uterine cervix, and has been reported to have 'precancerous' characteristics (1). Therefore, intraepithelial neoplasia has been used to indicate cancer precursors and lesions with high risk for cancer in certain types of adenocarcinomas, including those of the breast, pancreas and endometrium (2-4). The typical histological appearance of intraepithelial neoplasia is characterized by the cytological features of cancer, for which it is a precursor, with atypical cell proliferation in anatomical structures, including normal ducti and acini (1-5).

Prostatic intraepithelial neoplasia (PIN) is a well-defined entity, which exhibits cytological changes and the proliferation of secretory cells, features which are comparable to those of cancer cells within the prostatic ducti and acini (5-8). Although PIN is defined as low- or high-grade, low-grade is generally not considered relevant due to its low prognostic significance and low degree of interobserver agreement among pathologists $(7,9)$. Therefore, only the expression of high-grade PIN (HGPIN) was investigated in the present study. The acinar type of HGPIN is known to be closely associated with prostate adenocarcinoma, as HGPIN was reported to be observed in $85-100 \%$ of the materials examined following prostatectomies performed due to adenocarcinoma (9). In addition, a close association was reported between PIN and atypical glandular foci suspicious for malignancy (10). Furthermore, it was determined that the risk of malignancy increases in core prostate biopsies with the increased number of cores with PIN (11).

PIN has four basic architectural patterns: Tufting, micropapillary, cribriform and flat; in addition, other less common patterns, such as signet-ring cell, small-cell neuroendocrine, foamy, mucinous, squamous differentiation and inverted patterns have been reported (5-12). The inverted (hobnail) pattern is composed of secretory cell nuclei, which are histologically localized at the luminal surface of the prostate gland, rather than the periphery, and exhibit reverse 
polarity (12). The hobnail PIN pattern (variant) is a rare histological type in the prostate; at present, only one previous study has investigated this pattern, which evaluated a total of 15 cases of hobnail HGPIN (12). This inverted pattern was found to be primarily localized in the peripheral zone and was associated with adenocarcinoma in $45 \%$ of the cases (12).

The aim of the present study was to discuss the frequency of the hobnail PIN variant/pattern in core needle biopsies of prostate glands as well as to investigate the histological features and association with adenocarcinoma.

\section{Materials and methods}

Patients and histological samples. In the present study, a total of 2,034 prostate transrectal ultrasonographic (TRUS) biopsy samples were evaluated for the presence of the hobnail PIN pattern. The samples were evaluated at the Pathology Clinic of the Istanbul Education and Research Hospital (Istanbul, Turkey) between January 2010 and 2014. Out of the 2,034 TRUS biopsy samples, 13 cases of inverted HGPIN were identified. A total of 12 core biopsies were performed for each of the 13 patients. Tissue samples (4-6 $\mu \mathrm{m})$ were fixed in formalin, paraffin-embedded onto glass slides and stained with hematoxylin and eosin for histological evaluation. The slides were then examined under a light microscope (BW51; Olympus Corporation Tokyo, Japan). The following criteria were required for the positive diagnosis of hobnail PIN: Localization of the nuclei on the luminal surface of the prostate gland, rather than the periphery, which indicates reverse polarity in the proliferated cells of the acini and ducts; and observation of less prominent nucleoli in the nuclei of cells compared with adjacent non-inverted cell nuclei. Cases diagnosed with hobnail PIN were then categorized according to whether the PIN was pure or associated with other variants or prostate adenocarcinoma (13). In addition, the Gleason score (14) was determined for cases with prostatic adenocarcinoma accompanying hobnail PIN. The Gleason grading system is used to score the histological growth pattern and glandular differentiation of prostatic adenocarcinoma cells. It includes five basic grade patterns ranging from grade 1-5 (grade 1, well-differentiated tumor pattern; grade 5, no glandular differentiation). The final Gleason score, which ranges from $2-10$, is calculated by the addition of the two most common grades: the primary (most common) pattern and the secondary (second most common) pattern. Thus, the most well-differentiated tumors exhibit a Gleason score of 2, and the least-differentiated tumors exhibit a score of 10 (14). Written informed consent was obtained from all patients and the study was approved by the ethics committee of Istanbul Education and Research Hospital.

Immunohistochemical analysis. Immunohistochemical studies were performed for p63, 34ßE12 and $\alpha$-methylacyl-coenzyme A racemase (AMACR) for the differential diagnosis with adenocarcinoma in cases diagnosed with inverted PIN. Immunohistochemistry was conducted using the Benchmark XT staining system (Ventana Medical Systems, Inc., Tucson, AZ, USA) and antibodies against 34ßE12 (monoclonal mouse anti-human; cat. no. CM 127 AC; 1:50; Biocare Medical Inc., Concord, CA, USA), AMACR

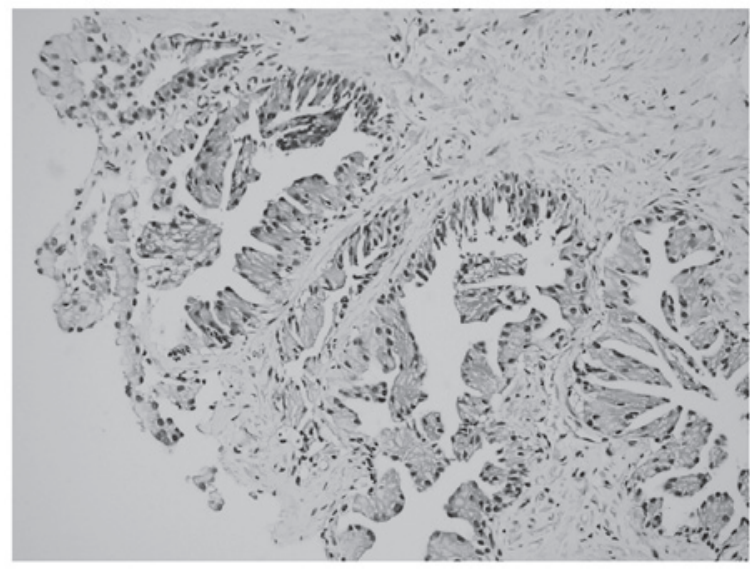

Figure 1. Representative low-power magnification image of inverted prostatic intraepithelial neoplasia demonstrating apical nuclei polarized toward the lumens (stain, hematoxylin and eosin; magnification, x200).

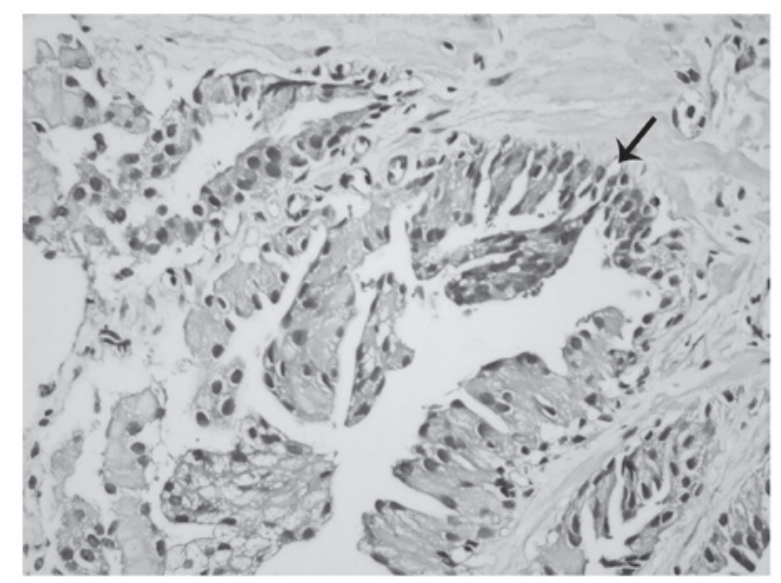

Figure 2. Representative high-power magnification image of inverted prostatic intraepithelial neoplasia demonstrating merging micropapillary (arrow) (stain, hematoxylin and eosin; magnification, x300).

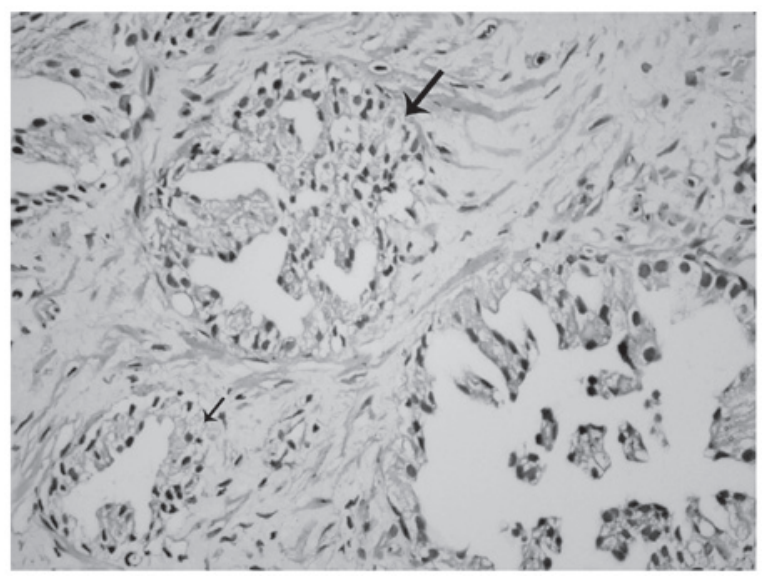

Figure 3. Representative image of inverted prostatic intraepithelial neoplasia showing a cribriform (large arrow) and flat (small arrow) pattern (stain, hematoxylin and eosin; magnification, x200).

(monoclonal rabbit anti-human; cat. no. 504S-14; 1:100; Cell Marque Corporation, Rocklin, CA, USA) and p63 (monoclonal mouse anti-human; cat. no. VP 163 GG25; 


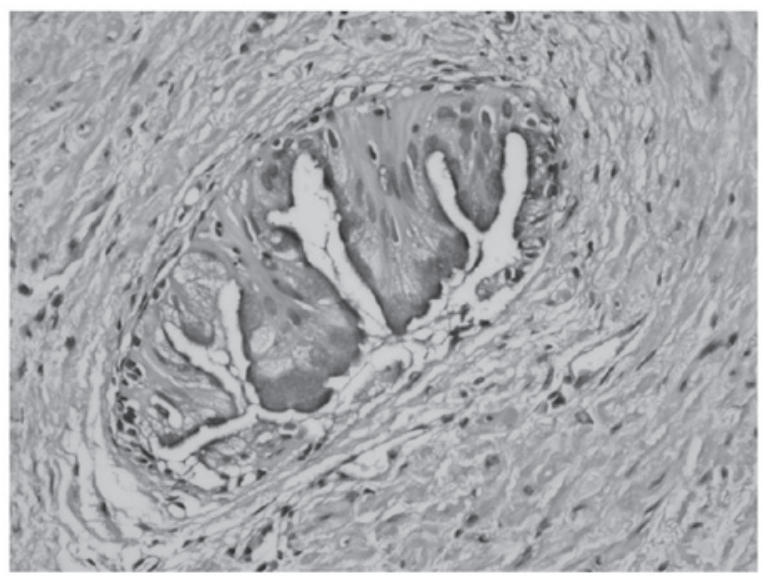

Figure 4. Representative image of inverted prostatic intraepithelial neoplasia with demonstrating apical nuclei with mucinous features (stain, hematoxylin and eosin; magnification, $\mathrm{x} 400)$.

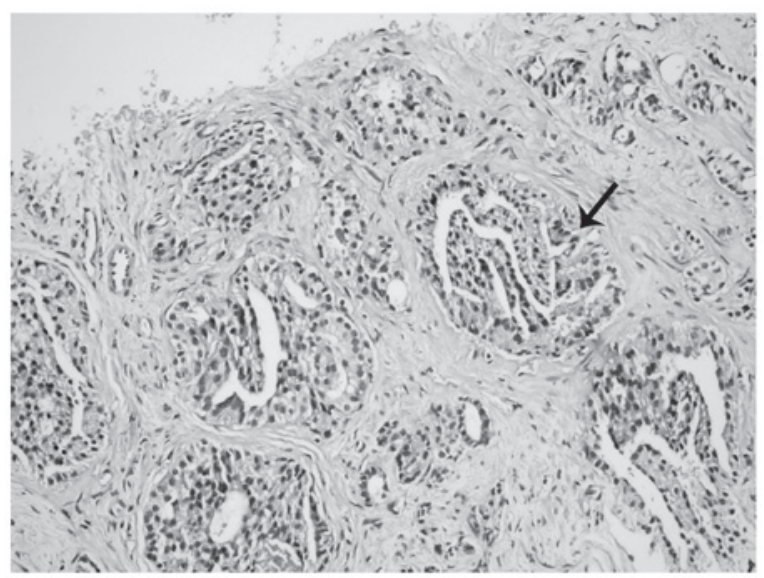

Figure 5. Representative image of inverted prostatic intraepithelial neoplasia with a cribriform pattern (arrow) (stain, hematoxylin and eosin; magnification, $\mathrm{x} 200)$.

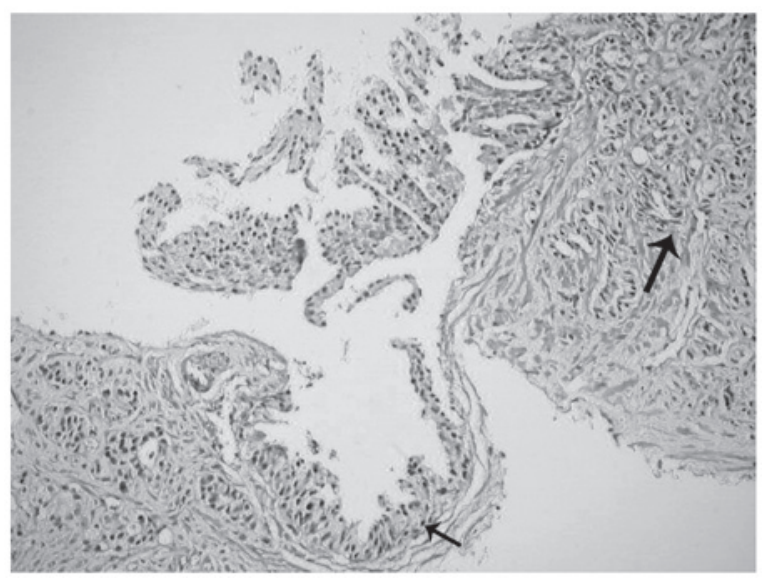

Figure 6. Representative image of inverted prostatic intraepithelial neoplasia (small arrow) associated with invasive prostatic adenocarcinoma (large arrow) (stain, hematoxylin and eosin; magnification, x200). Gleason score, $3+4=7$.

1:200; Biocare Medical Inc.). Briefly, the tissue sections were deparaffinized with EZ Prep solution (Ventana Medical

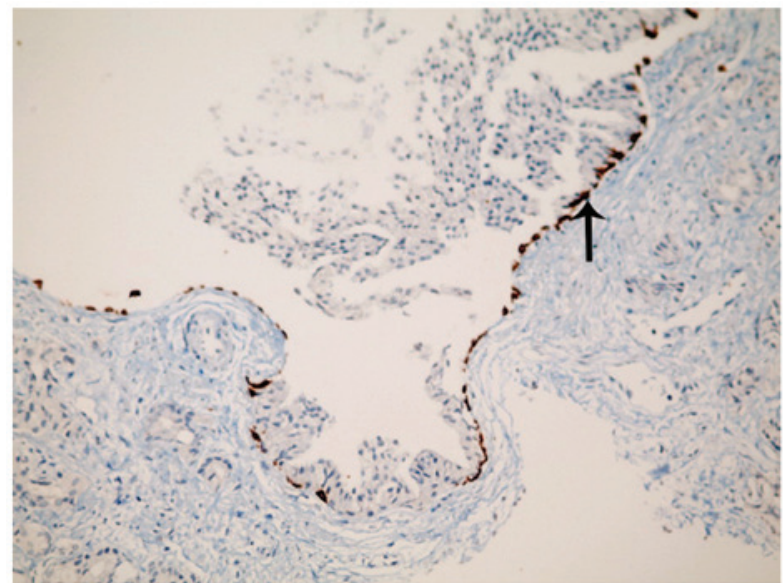

Figure 7. Representative image of the intact basal cell layer, as shown by high molecular weight cytokeratin (34ßE12) (arrow) (hematoxylin and eosin; magnification, $\mathrm{x} 200)$.

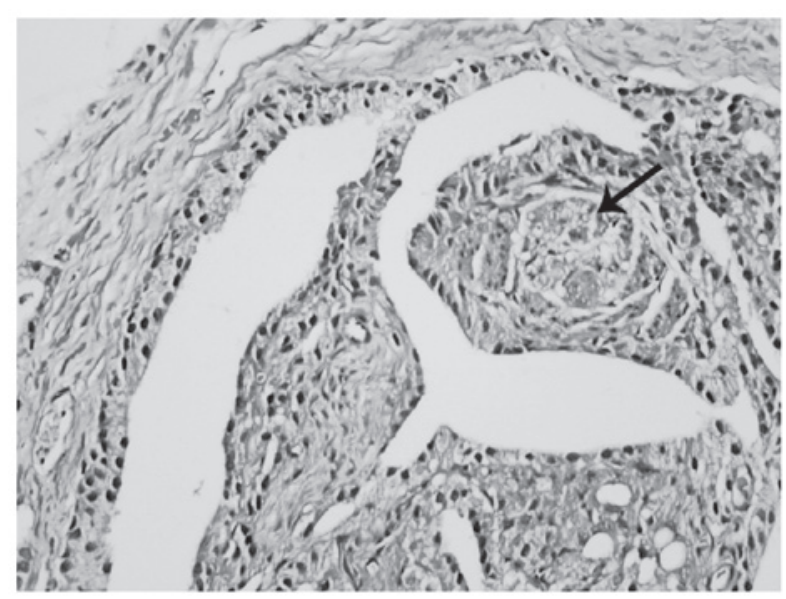

Figure 8. Representative image of a peripheral nerve (arrow) surrounded by adenocarcinoma cells with inverted features (stain, hematoxylin and eosin; magnification, $\mathrm{x} 400)$.

Systems, Inc.) at $75^{\circ} \mathrm{C}$, pretreated with cell conditioning 1 (CC1) solution (Ventana Medical Systems, Inc.) for antigen retrieval at $95^{\circ} \mathrm{C}$, and incubated with hydrogen peroxide (Ventana Medical Systems, Inc.) for 4 min to block endogenous peroxidase activity. The sections were then incubated with the AMACR, p63 and 34ßE12 primary antibodies for $32 \mathrm{~min}$ at $37^{\circ} \mathrm{C}$. Next, the sections were blocked using the Endogenous Biotin Blocking Kit (Ventana Medical Systems, Inc.) followed by incubation with a streptavidin-horseradish peroxiade-conjugated secondary antibody (monoclonal goat anti-rat; cat. no. 760-500; 1:200; Ventana Medical Systems, Inc.) for $8 \mathrm{~min}$ at $37^{\circ} \mathrm{C}$. The immunolocalized AMACR, p63 and $34 \beta E 12$ were visualized using a copper-enhanced DAB reaction. The slides were counterstained with hematoxylin II (Ventana Medical Systems, Inc.) for $4 \mathrm{~min}$ and Bluing Reagent (Ventana Medical Systems, Inc.) for $4 \mathrm{~min}$ and coverslips were applied using an automated coverslipper (Tissue-Tek Film Automated Coverslipper; Sakura Finetek Japan Co., Ltd., Tokyo, Japan). Cytoplasmic staining for AMACR and $34 \beta E 12$, and nuclear staining for p63 were considered positive. The clinical data of the patients, 
including age and medical history, were obtained from the patient charts. None of the cases involved in the present study with adenocarcinoma underwent surgery at the hospitals affiliated with the authors; therefore, radical prostatectomy materials were not examined.

\section{Results}

Identification of hobnail PIN. Out of the 2,034 biopsy samples that were examined in the present study, the hobnail PIN pattern was identified in a total of $13(0.63 \%)$ samples. The age range of the 13 patients was 53-83 years, with a mean age of 64 years. Hobnail PIN was observed in 4 cores in 3 patients, 2 cores in 5 patients and 1 core in 5 patients. In addition, prostatic acinar type adenocarcinoma was identified in 7 cases. The Gleason score was 7 in all of the adenocarcinoma cases.

Histological examinations of hobnail PIN. Proliferation in the form of micropapillary projections and mounds (tufting), particularly in the acini, was observed via microscopy in all 13 patients. However, nuclei localized on the luminal surface of the glands and not on the periphery (reverse polarity) were the most notable features (Fig. 1). In addition, inverted cell nuclei frequently demonstrated less prominent nucleoli compared with adjacent non-inverted cell nuclei (Fig. 2). Certain acini demonstrated the inverted feature in all parts, while others only partially demonstrated this feature (Fig. 2). Micropapillary projections and tufting patterns were also accompanied by cribriform and flat patterns in certain inverted PIN cases (Fig. 3). Furthermore, inverted morphology and intracytoplasmic mucin were observed in certain acini in one case (Fig. 4). Pure inverted histology was present in 4 of the 13 cases and PIN with other patterns was identified in 9 patients.

Gleason scores. All 7 carcinomas accompanied by inverted PIN were conventional acinar adenocarcinoma cases with a Gleason score of 7 . These scores were of $3+4$ in 6 cases and $4+3$ in the other case.

Invasive features of hobnail PIN associated with invasive adenocarcinoma. One case of acinar adenocarcinoma accompanying inverted PIN revealed large hobnail areas in the invasive component. The nuclei in the invasive cribriform structures had proliferated and were located on the luminal aspect of the cytoplasm, which was comparable to that of the inverted HGPIN sections. In addition, similar characteristics were observed in the separate invasive glands as well (Figs. 5-8). A cribriform pattern and single glandular structures were prominent in the invasive component (Fig. 5). Similar patterns were also observed surrounding inverted PIN areas (Fig. 6). Immunohistochemical 34ßE12 expression was evident along the basal cell layer in glands with inverted PIN (Fig. 7). Typical inverted features were also identified in tumoral glandular structures surrounding the peripheral nerves (Fig. 8).

Immunohistochemical analysis. Basal cell markers, p63 and $34 \beta E 12$, were found to be positively expressed in the hobnail PIN areas, indicating the presence of a basal cell layer and the absence of carcinoma. In addition, cytoplasmic staining with AMACR was observed in all 13 cases following immunohistochemical study, confirming the presence of both PIN and carcinoma.

\section{Discussion}

HGPIN requires much consideration due to its frequent association with prostate cancer in prostatic core biopsies; increased risk of cancer has been associated with an increased number of HGPIN-positive cores. In addition, at the molecular level, HGPIN displays the characteristics of a precursor cancerous lesion (5-12,15-17). PIN has four well-defined primary architectural patterns, including tufting, micropapillary, cribriform and flat, which do not carry prognostic significance (12). In addition, the signet-ring cell, small-cell neuroendocrine, foamy, mucinous, squamous differentiation and inverted patterns are less commonly observed (12).

The inverted (hobnail) pattern is rare and has only been reported by one previous study (12). Argani and Epstein (12) reported the incidence rate of hobnail PIN as $0.43 \%$ in their study. In the present study, an incidence rate of $0.63 \%$ was determined; however, the present study was prospective and actively searched for this entity in all TRUS biopsies, which may account for the increase in reported incidence rate.

A hobnail appearance is the typical feature of clear cell adenocarcinoma, which is predominantly observed in the genital system (18). The distinguishing features of hobnail from other patterns include the polarization of enlarged cell nuclei towards the lumen of the glands. This pattern may be missed as it is rare in prostate biopsies and resembles the central zone acini of the prostate; however, it may be distinguished from the central zone acini with features such as enlarged nuclei and with the known localization of the biopsy (12).

An association with prostate adenocarcinoma was identified in almost half of the cases in a previous study on inverted PIN (12). In the present study, it was observed that 7 of the 13 cases were associated with acinar adenocarcinoma. The Gleason score was 7 in all the adenocarcinoma-associated cases in the present study, which was higher than those reported in the Argani and Epstein study: The Gleason score was $6(3+3)$ in $80 \%$ of the cases with adenocarcinoma accompanying PIN in the Argani and Epstein (12) study of 15 cases. The high association with carcinoma and high Gleason score may therefore have interesting prognostic significance for inverted PIN.

In the present study, histological features of conventional prostatic acinar adenocarcinoma were identified in 6 of the 7 cases with adenocarcinoma accompanying hobnail PIN. The remaining case exhibited a different adenocarcinoma histology and demonstrated features similar to that of the accompanying hobnail PIN. Therefore, it was difficult to distinguish the invasive carcinoma cribriform pattern areas with PIN morphology from the actual hobnail PIN regions in certain areas. The negative immunohistochemical results of the basal cell markers, p63 and 34ßE12, in the carcinoma areas and positive results in the hobnail PIN areas aided the differential diagnosis. In addition, most of the individual invasive glands outside the cribriform pattern demonstrated inverted features. The Gleason score of this case was $7(4+3)$ and tumor 
was present in all 12 core biopsies. In a study by Arkani and Epstein (12), no adenocarcinoma cases with inverted features were reported. Thus, to the best of our knowledge, the current study reports the first case of prostatic adenocarcinoma with hobnail histological features in the invasive component in the English literature.

In conclusion, the present study identified the histological features of the uncommonly diagnosed hobnail PIN. The high rate of association with prostatic acinar adenocarcinoma (54\%) and the high Gleason score (7) of the carcinomas were noteworthy. However, further studies are required in order to determine whether the hobnail pattern is aggressive. In addition, the present study identified inverted pattern histological features in the invasive component of an associated adenocarcinoma in one case of hobnail PIN.

\section{References}

1. Kurman RJ, Malkasian GD Jr, Sedlis A and Solomon D: From Papanicolaou to Bethesda: The rationale for a new cervical cytologic classification. Obstet Gynecol 77: 779-782, 1991.

2. Hruban RH, Adsay NV, Albores-Saavedra J, et al: Pancreatic intraepithelial neoplasia: A new nomenclature and classification system for pancreatic duct lesions. Am J Surg Pathol 25: 579-586, 2001.

3. Mutter GL: Histopathology of genetically defined endometrial precancers. Int J Gynecol Pathol 19: 301-309, 2000.

4. Rosai J: Borderline epithelial lesions of the breast. Am J Surg Pathol 15: 209-221, 1991.

5. Bostwick DG: Progression of prostatic intraepithelial neoplasia to early invasive adenocarcinoma. Eur Urol 30: 145-152, 1996.

6. Epstein JI and Herawi M: Prostate needle biopsies containing prostatic intraepithelial neoplasia or atypical foci suspicious for carcinoma: Implications for patient care. J Urol 175 (3 Pt 1): 820-834, 2006.

7. Zhou M, Netto JG and Epstein JI (eds): Neoplastic disease of the prostate. In: High-Yield Pathology: Uropathology. Elsevier, Philadelphia, PA, pp52-54, 2012.
8. Bostwick DG, Liu L, Brawer MK and Qian J: High-grade prostatic intraepithelial neoplasia. Rev Urol 6: 171-179, 2004.

9. Cheng L, Paterson RF, Beck SD and Parks J: Prostatic intraepithelial neoplasia: An update. Clin Prostate Cancer 3: 26-30, 2004.

10. Cheville JC, Reznicek MJ and Bostwick DG: The focus of 'atypical glands, suspicious for malignancy' in prostatic needle biopsy specimens: Incidence, histologic features, and clinical follow-up of cases diagnosed in a community practice. Am J Clin Pathol 108: 633-640, 1997.

11. Kronz JD, Allan CH, Shaikh AA and Epstein JI: Predicting cancer following a diagnosis of high-grade prostatic intraepithelial neoplasia on needle biopsy: Data on men with more than one follow-up biopsy. Am J Surg Pathol 25: 1079-1085, 2001.

12. Argani P and Epstein JI: Inverted (Hobnail) high-grade prostatic intraepithelial neoplasia (PIN): Report of 15 cases of a previously undescribed pattern of high-grade PIN. Am J Surg Pathol 25: 1534-1539, 2001

13. Elbe JN, Sauter G, Epstein JI and Sesterhenn IA (eds): Tumours of the prostate. In: World Health Organization Classification of Tumours. Pathology and Genetics of Tumours of the Urinary System and Male Genital Organs. IARC Press, Lyon, France, 159-214, 2004

14. Epstein JI, Allsbrook WC Jr, Amin MB and Egevad LL; ISUP Grading Committee: The 2005 International Society of Urological Pathology (ISUP) Consensus Conference on Gleason Grading of Prostatic Carcinoma. Am J Surg Pathol 29: 1228-1242, 2005 .

15. Hughes C, Murphy A, Martin C, Sheils O and O'Leary J: Molecular pathology of prostate cancer. J Clin Pathol 58: 673-684, 2005.

16. Godoy G and Taneja SS: Contemporary clinical management of isolated high-grade prostatic intraepithelial neoplasia. Prostate Cancer Prostatic Dis 11: 20-31, 2008.

17. Merrimen JL, Evans AJ and Srigley JR: Preneoplasia in the prostate gland with emphasis on high grade prostatic intraepithelial neoplasia. Pathology 45: 251-263, 2013.

18. Montag AG, Jenison EL, Griffiths CT, Welch WR, Lavin PT and Knapp RC: Ovarian clear cell carcinoma. A clinicopathologic analysis of 44 cases. Int J Gynecol Pathol 8: 85-96, 1989. 